\title{
ESTUDO DA ADSORÇÃO DE Cu(II) UTILIZANDO CASCA DE EUCALIPTO COMO BIOSSORVENTE
}

\author{
Tiago Guimarães ${ }^{1}$ \\ Yago Ricardo de Oliveira ${ }^{2}$ \\ D'avila Leal Polastreli ${ }^{3}$ \\ Cristiane Francisca Barbosa ${ }^{4}$ \\ Ronaldo Willian da Silva ${ }^{5}$ \\ Renato Ribeiro Passos ${ }^{6}$ \\ Luciene Paula Roberto Profeti ${ }^{7}$ \\ Demetrius Profeti ${ }^{8}$
}

Resumo: Neste trabalho foi investigada a aplicabilidade de um adsorvente de baixo custo, a casca de eucalipto (CE), para a remoção de íons Cu (II) de soluções aquosas. A casca de eucalipto foi pirolisada a $600^{\circ} \mathrm{C}$ e aplicada tanto na forma de pó como na forma não pulverizada. Além disso, verificou-se o efeito do tempo de contato do adsorvato com o material adsorvente, determinando-se a concentração de íons cobre após o tempo de adsorção. Os resultados obtidos mostraram a potencialidade do uso da CE pirolisada e pulverizada como material biossorvente.

Palavras-chave: Adsorvato; Metais pesados; Adsorção; Casca de eucalipto.

\footnotetext{
1 Programa de Pós-Graduação em Agroquímica/Universidade Federal do Espírito Santo, Brasil. E-mail: thiago.vegetta@hotmail.com.

2 Programa de Pós-Graduação em Agroquímica/Universidade Federal do Espírito Santo, Brasil. E-mail: yagordeoliveira@gmail.com.

${ }_{3}$ Programa de Pós-Graduação em Agroquímica/Universidade Federal do Espírito Santo, Brasil. E-mail: davilapolastreli@hotmail.com.

${ }_{4}$ Programa de Pós-Graduação em Agroquímica/Universidade Federal do Espírito Santo, Brasil. E-mail: crisfbsp@gmail.com.

5 Programa de Pós-Graduação em Agroquímica/Universidade Federal do Espírito Santo, Brasil. E-mail: ronaldo_willian1@hotmail.com.

6 Programa de Pós-Graduação em Agroquímica/Universidade Federal do Espírito Santo, Brasil. E-mail: renatoribeiropassos@hotmail.com.

7 Programa de Pós-Graduação em Agroquímica/Universidade Federal do Espírito Santo, Brasil. E-mail: Iprprofeti@gmail.com.

8 Programa de Pós-Graduação em Agroquímica/Universidade Federal do Espírito Santo, Brasil. E-mail: dprofeti@gmail.com.
} 